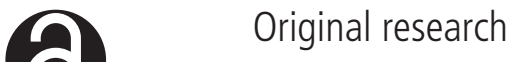

The impact of software-based metal artifact reduction OPEN ACCESS

\section{on the liquid embolic agent Onyx in cone-beam CT: a systematic in vitro and in vivo study}

\author{
Niclas Schmitt (1) , ${ }^{1}$ Charlotte S Weyland (10) , Lena Wucherpfennig (1) , \\ Christof M Sommer, ${ }^{2,3,4}$ Martin Bendszus, ${ }^{1}$ Markus A Möhlenbruch, ${ }^{1}$ \\ Dominik F Vollherbst (1) ${ }^{1}$
}

- Additional supplemental material is published online only. To view, please visit the journal online (http://dx.doi. org/10.1136/neurintsurg2021-018018)

'Department of Neuroradiology, Heidelberg University Hospital, Heidelberg, Germany ${ }^{2}$ Department of Diagnostic and Interventional Radiology, Heidelberg University Hospital, Heidelberg, Germany ${ }^{3}$ Department of Nuclear Medicine, Heidelberg University Hospital, Heidelberg, Germany ${ }^{4}$ Clinic of Radiology and Neuroradiology, Sana Kliniken Duisburg, Duisburg, Germany

Correspondence to Dr Dominik F Vollherbst, Department of Neuroradiology, Heidelberg University Hospital, INF 400, 69120 Heidelberg, Germany; dominik.vollherbst@ med.uni-heidelberg.de

Received 10 July 2021 Accepted 15 August 2021

\section{Check for updates}

(c) Author(s) (or their employer(s)) 2021. Re-use permitted under CC BY-NC. No commercial re-use. See rights and permissions. Published by BMJ.

\section{To cite: Schmitt N Weyland CS, \\ Wucherpfennig L, et al. J Neurolntervent Surg Epub ahead of print: [please include Day Month Year]. doi:10.1136/ \\ neurintsurg-2021-018018}

\section{ABSTRACT}

Background Onyx is frequently used for endovascular embolization of intracranial arteriovenous malformations (AVMs) and dural arteriovenous fistulas (dAVFs). One drawback of using Onyx is the generation of artifacts in cone-beam CT (CBCT). These artifacts can represent an obstacle for the detection of periprocedural hemorrhage or planning of subsequent radiosurgery. This study investigates the effect of artifact reduction by the syngo DynaCT SMART Metal Artifact Reduction (MAR) software. Methods A standardized in vitro tube model $(n=10)$ was filled with Onyx 18 and CBCT image acquisition was conducted in a brain imaging phantom. Furthermore, post-interventional CBCT images of 20 patients with AVM $(n=13)$ or dAVF $(n=7)$, each treated with Onyx, were investigated. The MAR software was applied for artifact reduction. Artifacts of the original and the post-processed images were analyzed quantitatively (standard deviation in a region of interest on the layer providing the most artifacts) and qualitatively. For the patient images, the effect of the MAR software on brain parenchyma on artifact-free images was further investigated.

Results Quantitative and qualitative analyses of both datasets demonstrated a lower degree of artifacts in the post-processed images (eg, patient images: $38.30 \pm 22.03$ density units (no MAR; mean SD $\pm S D$ ) vs $19.83 \pm 12.31$ density units (with MAR; $p<0.001$ ). The MAR software had no influence on the brain parenchyma in artifact-free images.

Conclusion The MAR software significantly reduced the artifacts evoked by Onyx in CBCT without affecting the visualization of brain parenchyma on artifact-free images. Applying this software could thus improve the quality of periprocedural CBCT images after embolization with Onyx.

\section{INTRODUCTION}

Endovascular embolization using liquid embolic agents (LEAs) is an effective treatment option for cerebral arteriovenous malformations (AVMs) and dural arteriovenous fistulas (dAVFs). ${ }^{1-3}$ Despite the increasing number of different commercially available LEAs, the embolic agent Onyx (Medtronic, Irvine, USA) is still one of the most commonly used products. ${ }^{2}{ }^{4}$ Onyx is a non-adhesive agent, consisting of ethylene vinyl alcohol (EVOH) copolymer, dimethyl sulfoxide (DMSO) and radiopaque tantalum powder. One frequently reported drawback of Onyx is the generation of artifacts in CT and cone-beam CT (CBCT) ${ }^{5-8}$ Since cerebral AVMs and dAVFs have an increased risk of periprocedural bleeding, the artifacts induced by Onyx may represent a major obstacle for the detection of acute intracranial hemorrhage. ${ }^{9}{ }^{10}$ Besides, detection of residual nidus in large, incompletely embolized AVMs or dAVFs is hampered by artifacts. Furthermore, especially complex AVMs may not be occluded completely by endovascular means and often require additional radiation therapy or microneurosurgery. ${ }^{3}$ For stereotactic radiosurgery, CBCT images of the vascular malformation are often a major component of the planning of the radiation treatment. In this regard, the LEA-induced artifacts can represent a further obstacle for an effective and tissue-conserving radiation treatment planning. ${ }^{11-15}$

CBCT enables a CT-like visualization of the target region during and after the endovascular intervention. ${ }^{16} \mathrm{CBCT}$ equipped angiography systems have therefore the potential to assess periprocedural complications, such as acute intracranial hemorrhage or hydrocephalus, within the angiography suite. ${ }^{17}$ To improve the image quality and reduce metal-related artifacts, several acquisition and postprocessing techniques have been introduced over the past years. ${ }^{18}$ One frequently used application is the syngo DynaCT SMART software (Siemens Healthineers, Erlangen, Germany; hereinafter referred to as MAR (metal artifact reduction)), which is a commercially available add-on software for the angiography systems ARTIS icono, ARTIS pheno, ARTIS Q and ARTIS zee (Siemens Healthineers, Erlangen, Germany, respectively). Several studies have demonstrated its ability to reduce metal-related artifacts, for example, after intracranial coil embolization, while studies investigating artifact reduction caused by embolic agents are rare. ${ }^{19-24}$ The aim of the present study was to provide a systematic analysis on the effect of MAR software for the reduction of artifacts evoked by Onyx in a standardized in vitro model and in postinterventional patient CBCT images.

\section{MATERIALS AND METHODS}

\section{Sample preparation and data collection}

A standardized two-dimensional in vitro tube model was used for investigation, as described previously. ${ }^{6}$ 
In detail, straight-configured, DMSO-compatible tubes with an outer diameter of $8 \mathrm{~mm}$, an inner diameter of $4 \mathrm{~mm}$ and a length of $30 \mathrm{~mm}$ were created, as these dimensions are comparable to intracranial human vessels. ${ }^{25}$ The models were flushed with warm saline $\left(38.0^{\circ} \mathrm{C}\right.$, sodium chloride $\left.0.9 \%\right)$ before filling, and the embolic agent Onyx 18 was prepared in accordance with the manufacturer's instructions. In the next step, each tube was completely filled with Onyx 18 by manual pulsatile injection with an average flow of $0.5 \mathrm{~mL}$ per minute and inserted into a custom-made CT-phantom with an average density similar to brain tissue, following the model of Daubner et al. ${ }^{26}$ Ten standardized in vitro models, each filled with a total volume of $3.78 \mathrm{~mL}$, were investigated.

The in vivo datasets were collected retrospectively using a prospectively maintained patient database. Approval and informed consent for data collection were provided by the local ethics committee and by the patients, respectively. All cases of patients who underwent endovascular embolization of an intracranial AVM or dAVF using Onyx and received a post-procedural CBCT in our department between January 2016 and May 2021 were included. In addition, patients who received small-volume injections of cyanoacrylates (Glubran 2, GEM SRL, Viareggio, Italy), mixed with iodized oil (Lipiodol Ultra-Fluid, Guerbet, Villepinte, France), distant to the Onyx cast (defined as a distance of $\geq 1 \mathrm{~cm}$ in the $\mathrm{z}$-axis) were also included, whereas patients who were embolized with other copolymer-based LEAs were excluded from this study. Further exclusion criteria were the absence of a post-procedural CBCT or a treatment before 2016, because an older angiography system was used. The screening phase involved a review of the reports and a visual inspection of all corresponding CBCT images. Twenty patients matched the inclusion criteria.

\section{Imaging and image post-processing}

Image acquisition of all CBCT images was performed on a biplanar angiography system (Artis Q, Siemens Healthineers, Erlangen, Germany) with standard settings according to clinical routine: $20 \mathrm{~s}$ rotational acquisition generating 500 projections with an angular step of $0.4^{\circ}$ for a total coverage of $200^{\circ}$ with a pulse length of $12.5 \mathrm{~ms}$, a tube voltage of $109 \mathrm{kV}$ and a dose per frame of $1.82 \mu \mathrm{Gy}$. In order to generate images with artifact reduction, the MAR software was applied to the original images of the in vitro and in vivo datasets. Therefore, the raw data of each dyna CT scan has to be uploaded to the MAR software platform while for each scan the process of artifact reduction must be applied manually. Afterwards, the original and the postprocessed images were reconstructed in the axial plane with a slice thickness of $4 \mathrm{~mm}$.

\section{Quantitative image analysis}

A schematic description of the quantitative analysis is illustrated in figure 1. Quantitative analysis of the in vitro tube models was performed using the Medical Imaging Interaction Toolkit (MITK; German Cancer Research Center (DKFZ), Heidelberg, Germany). ${ }^{27}$ As described previously, the MITK software allowed us to place a highly standardized region of interest (ROI) with a donut-shaped configuration adjacent to and surrounding the center of each in vitro model. ${ }^{56}$ For each in vitro model, a donut-shaped ROI with an inner diameter of $5 \mathrm{~mm}$ and an outer diameter of $20 \mathrm{~mm}$ was placed on the central image slice of the upper third, the middle third and the lower third, respectively. This procedure ensured that artifacts of different positions were taken into account.

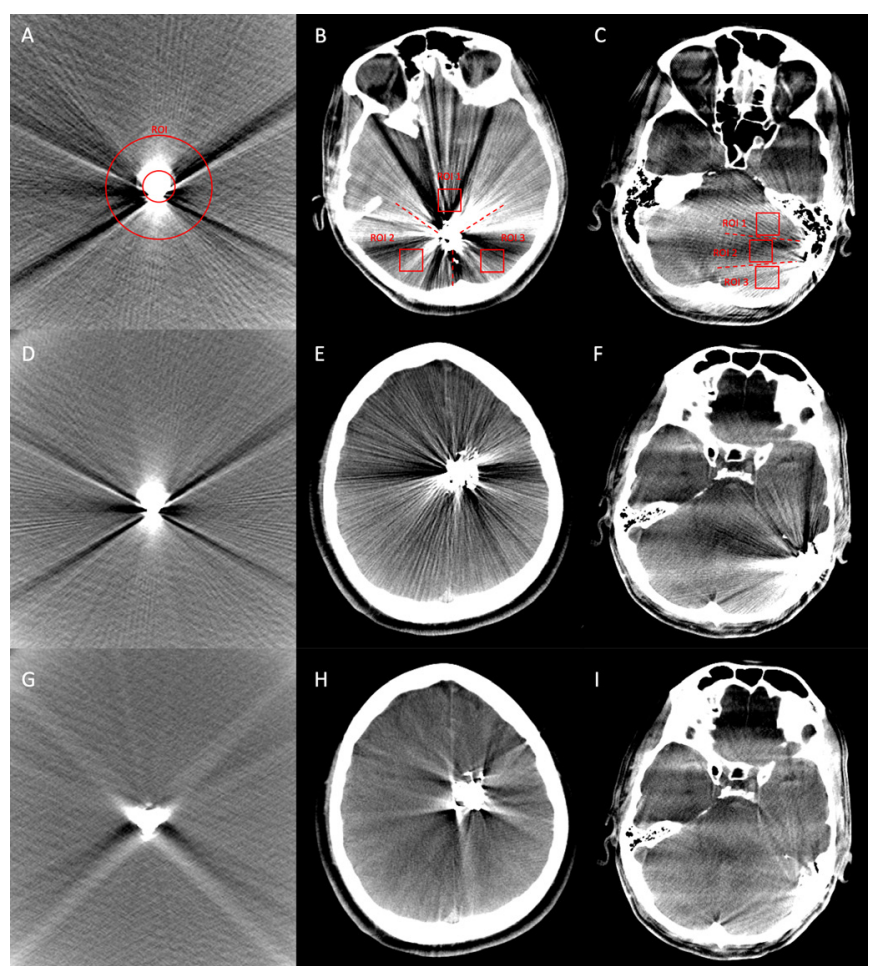

Figure 1 Illustration of the quantitative image analysis of the in vitro tube models, using a donut-shaped ROI (A), and of the patient data, using three square shaped ROls, which were drawn manually into the center of each third of the surrounding sector ( $B$, patient with AVM; $C$, patient with $d A V F$ ). The identical procedure was performed on the postprocessed images (with MAR). Comparing the same image slices of the original (no MAR, D-F) and the post-processed (with MAR, G-I) CBCT images, a significant reduction of the Onyx-induced artifacts by the MAR software could be observed ( $D$ and $G$, tube model; $E$ and $H, A V M$ located in the left frontal lobe; $F$ and I, dAVF located at the left sigmoid sinus). AVM, arteriovenous malformation; CBCT, cone-beam CT; dAVF, dural arteriovenous fistula; MAR, metal artifact reduction; ROI, region of interest.

For the quantitative analysis of the patient data, a picture archiving and communication system workstation (CENTRICITY PACS 4.0; GE Healthcare, Barrington, IL, USA) was used. Therefore, a standardized ROI was drawn manually in consensus with a neuroradiology resident and a neuroradiology attending (5 years and 8 years of experience in diagnostic imaging, respectively) on the image layer providing the most LEA-related artifacts in the original images. Originating from the LEA cast and the individual localization of the vascular malformation, the surrounding brain parenchyma was divided into a roundish (mostly for the AVMs) or a fan-shaped (for the dAVFs) sector, while for each sector thirds of similar size were defined. A square shaped ROI with a side length of $20 \mathrm{~mm}$ each was drawn manually into the center of each third. The distance between ROI and the LEA cast was $15 \mathrm{~mm}$, respectively. On a second monitor, a ROI of equal configuration and similar position was placed manually in the post-processed CBCT images.

Additional analyses were performed for the post-interventional CBCT images of all 20 embolized patients. Three ROIs of similar configuration (side length of $10 \mathrm{~mm}$ each) were manually drawn into brain parenchyma on a layer which was not affected by LEA-related artifacts in the original images. Three ROIs of the same configuration and similar localization were also placed in the post-processed CBCT images. 
Since LEA-related artifacts usually consist of areas of high and low density, the degree of artifacts was assessed by calculating the standard deviation (SD) of the density units (DU). ${ }^{5-8} 14$

\section{Qualitative image analysis}

Qualitative analysis of the degree of artifacts in the CBCT images of the in vitro and in vivo datasets was performed by two different readers (reader 1 with 5 years and reader 2 with 8 years of experience in diagnostic imaging) on a CENTRICITY PACS 4.0 workstation. Each reader was blinded to the type of image reconstruction: no MAR versus with MAR; for the in vivo datasets to the type of vascular malformation as well. Both readers were allowed to adjust the window manually. The degree of LEA-induced artifacts was graded by a five-point scale: (1) severe artifacts, largely not diagnostic; (2) poor image quality, partly non-diagnostic; (3) moderate image quality, limited diagnostic confidence; (4) good image quality, sufficient for diagnosis; (5) excellent image quality, no artifacts. ${ }^{8}$

Further analyses were performed for the post-interventional in vivo $\mathrm{CBCT}$ images. Both readers assessed the differentiation of the gray and white matter on the image layers which were not affected by LEA-related artifacts by a five-point scale: (1) not differentiable, $<10 \%$; (2) poorly differentiable, $11-30 \%$; (3) moderately differentiable, 31-60\%; (4) well differentiable, 61-90\%; (5) completely differentiable, $>90 \%$.

\section{Statistics}

GraphPad Prism software (version 9.1.0, La Jolla, CA, USA) was used for statistical analysis. Data of the quantitative analysis are presented as mean SD \pm SD and of the qualitative analysis as mean \pm SD. To evaluate statistical differences between the original and the post-processed CBCT images, the Wilcoxon matched-pairs signed rank test was conducted. The level of statistical significance was defined as $\mathrm{p}<0.05$.

For qualitative analyses, further assessment of the inter-reader agreement by using the Cohen's $\kappa$ coefficient was performed. ${ }^{28}$ The $\kappa$ values were interpreted as follows: $\leq 0.20$, no agreement; 0.21-0.39, minimal agreement; 0.40-0.59, weak agreement; $0.60-0.79$, moderate agreement; $0.80-0.90$, strong agreement; and $\geq 0.90$, almost perfect agreement. ${ }^{29}$

\section{RESULTS}

Representative CBCT images of the original and the postprocessed images of the standardized tube model and postinterventional patient data are demonstrated in figure 1.

The quantitative results are summarized in table 1 and illustrated in figure 2 and the qualitative results are summarized in table 2 and illustrated in figure 2 .

Onyx 18 was the applied concentration in all embolizations.

Comparing the artifacts induced by Onyx 18 on the same image layers of the original and the post-processed CBCT images of the standardized in vitro tube models, the Wilcoxon matchedpairs signed rank test showed a significantly lower degree of artifacts in the post-processed images in quantitative (no MAR: 56.36 \pm 25.90 DU vs with MAR: 42.01 \pm 6.96 DU; p<0.001) and qualitative analysis (no MAR: $1.00 \pm 0.00 \mathrm{vs}$ with MAR: $3.50 \pm 0.51 ; \mathrm{p}<0.001)$.

In our institutional patient database, 20 patients met the inclusion criteria, while 13 patients with AVM and seven with dAVF were included. A more detailed description of the individual patient and procedural characteristics is provided in the online supplemental appendix. The total volume of the LEA used for
Table 1 Summary of the results of the quantitative image analysis of the in vitro and in vivo datasets

\begin{tabular}{llll}
\hline & $\begin{array}{l}\text { Original CBCT images } \\
\text { (no MAR)* }\end{array}$ & $\begin{array}{l}\text { Post-processed CBCT images } \\
\text { (with MAR) }\end{array}$ & P valuest \\
\hline Tubes & $56.36 \pm 25.90 \mathrm{DU}$ & $\begin{array}{l}42.01 \pm 6.96 \mathrm{DU} \\
19.83 \pm 12.31 \mathrm{DU}\end{array}$ & $\mathrm{p}<0.001$ \\
$\begin{array}{l}\text { Patients } \\
\text { AVMs+dAVFs }\end{array}$ & $38.30 \pm 22.03 \mathrm{DU}$ & $17.95 \pm 12.72 \mathrm{DU}$ & \\
$\begin{array}{l}\text { Patients } \\
\text { AVMs }\end{array}$ & $37.82 \pm 20.31 \mathrm{DU}$ & $23.33 \pm 10.96 \mathrm{DU}$ & \\
$\begin{array}{l}\text { Patients } \\
\text { dAVFs }\end{array}$ & $39.19 \pm 25.43 \mathrm{DU}$ & $8.11 \pm 1.54 \mathrm{DU}$ & $\mathrm{p}=0.908$ \\
\hline $\begin{array}{l}\text { Patients } \\
\text { Artifact-free layer }\end{array}$ & $8.12 \pm 1.39 \mathrm{DU}$ & & \\
\hline
\end{tabular}

The MAR software was able to reduce the LEA-related artifacts in CBCT significantly in the standardized in vitro models and the patient datasets in the quantitative analysis. There was no difference between the original and the post-processed images on the layers which were not affected by Onyx-related artifacts.

${ }^{*}$ Data are provided as mean SD \pm SD.

tP values of the Wilcoxon matched-pairs signed rank test to evaluate statistical differences between the original (no MAR) and the post-processed (with MAR) CBCT images.

AVM, arteriovenous malformation; CBCT, cone-beam CT; dAVF, dural arteriovenous fistula DU, density units; LEA, liquid embolic agent; MAR, metal artifact reduction.

each embolization procedure was not provided in the reports and could therefore not be analyzed.

The MAR software was able to significantly reduce the LEA-related artifacts in the post-interventional CBCT images of all 20 patients with AVM or dAVF quantitatively (no MAR: $38.30 \pm 22.03$ DU vs with MAR: 19.83 \pm 12.31 DU; $\mathrm{p}<0.001$ ) and qualitatively (no MAR: $1.00 \pm 0.00$ vs with MAR: $2.85 \pm 0.70 ; \mathrm{p}<0.001)$.

Similar results with significant reduction of the artifacts were also observed for the selective evaluation of the patients suffering from an AVM (quantitative: no MAR: 37.82 \pm 20.31
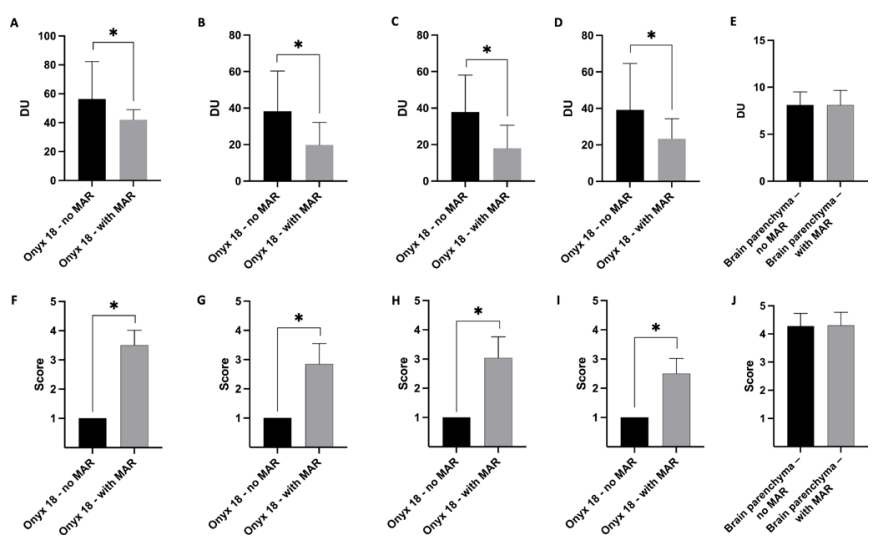

Figure 2 Illustration of the results of the quantitative and qualitative image analysis. The artifacts caused by the non-adhesive embolic agent Onyx 18 were significantly lower in the post-processed (with MAR) CBCT images in quantitative (A-D) and qualitative ( $F-I)$ analysis. There was no difference on the layer which was free of Onyx-related artifacts in quantitative (E: standard deviation of density units in a defined region of interest) and qualitative (J: differentiation of the gray and white matter using a five-point scale) analysis. Upper row, quantitative analysis; lower row, qualitative analysis; bars, mean; whiskers, SD. A and $F$, tube models; $B$ and $G$, all patients with AVM or dAVF; $C$ and $H$, patients with AVM; $D$ and I, patients with dAVF; $E$ and J, analysis of the brain parenchyma on the layer free of Onyx-related artifacts. *Statistically significant difference $(p<0.05)$. AVM, arteriovenous malformation; $C B C T$, cone-beam CT; dAVF, dural arteriovenous fistula; DU, density units; MAR, metal artifact reduction. 
Table 2 Summary of the results of the qualitative image analysis of the in vitro and in vivo datasets

\begin{tabular}{llll}
\hline & $\begin{array}{l}\text { Original CBCT images } \\
\text { (no MAR) }\end{array}$ & $\begin{array}{l}\text { Post-processed CBCT images } \\
\text { (with MAR) }\end{array}$ & P value* \\
\hline $\begin{array}{l}\text { Tubes } \\
\text { Patients }\end{array}$ & $1.00 \pm 0.00$ & $3.50 \pm 0.51$ & $\mathrm{p}<0.001$ \\
$\begin{array}{l}\text { AVMs+dAVFs } \\
\text { Patients }\end{array}$ & $1.00 \pm 0.00$ & $2.85 \pm 0.70$ & \\
$\begin{array}{l}\text { AVMs } \\
\text { Patients dAVFs }\end{array}$ & $1.00 \pm 0.00$ & $3.04 \pm 0.72$ & \\
$\begin{array}{l}\text { Patients } \\
\text { Artifact-free layer }\end{array}$ & $4.28 \pm 0.45$ & $2.50 \pm 0.52$ & \\
\hline
\end{tabular}

Five-point scale analysis of the in vitro and in vivo CBCT datasets by two different readers (shown as mean \pm SD). The Wilcoxon matched-pairs signed rank test demonstrated a significant reduction of Onyx-related artifacts by the MAR software in the CBCT images of the in vitro and in vivo datasets. Qualitative analysis showed that gray and white matter were completely differentiable on the image layers which were not affected by Onyx-related artifacts in the original (no MAR) and post-processed (with MAR) CBCT images.

${ }^{*} P$ values of the Wilcoxon matched-pairs signed rank test to evaluate statistical difference between the original (no MAR) and the post-processed (with MAR) CBCT images.

$A V M$, arteriovenous malformation; $C B C T$, cone-beam $C T$; $d A V F$, dural arteriovenous fistula; MAR, metal artifact reduction.

DU vs with MAR: $17.95 \pm 12.72 \mathrm{DU}$; qualitative: no MAR: $1.00 \pm 0.00$ vs with MAR: $3.04 \pm 0.72 ; \mathrm{p}<0.001$, respectively) or a dAVF (quantitative: no MAR: $39.19 \pm 25.43$ DU vs with MAR: $23.33 \pm 10.96$ DU; qualitative: no MAR: $1.00 \pm 0.00 \mathrm{vs}$ with MAR: $2.50 \pm 0.52 ; \mathrm{p}<0.001$, respectively).

The Wilcoxon matched-pairs signed rank test revealed no difference between the original and the post-processed images in the quantitative $(p=0.908)$ and the qualitative analyses $(p>0.999)$ on the layers which were initially not affected by LEA-related artifacts.

The inter-reader reliability showed a strong agreement for the in vitro $(\kappa=0.841$; range $0.633-1.00)$ and an almost perfect agreement for the in vivo $(\kappa=0.915$; range $0.843-$ 0.987 ) datasets for the qualitative analyses by both readers.

\section{DISCUSSION}

In this study, a systematic analysis of the effect of the syngo DynaCT SMART software on artifacts produced by Onyx in CBCT was performed in a standardized in vitro tube model and in post-interventional CBCT images of 20 patients with dAVFs or AVMs. The MAR software was able to reduce the Onyxrelated artifacts significantly, both in the in vitro model and in the patient imaging datasets, without affecting the visualization of the brain parenchyma distant to the Onyx cast in artifact-free CBCT images.

Several recently published preclinical studies compared the degree of artifacts of different LEAs in conventional CT and CBCT. $^{5-8}$ They demonstrated that the non-adhesive agent Onyx, which is currently one of the most frequently used LEAs for the treatment of cerebral vascular malformations, induces the highest degree of artifacts compared with other LEAs in both imaging modalities. ${ }^{5-8}$ We therefore focused our study on Onyx.

One of these studies showed that cyanoacrylates mixed with iodized oil (ratio 1:1) generated only minor artifacts in comparison to the other LEAs. ${ }^{6}$ For this reason, we assumed that the artifacts generated by cyanoacrylates mixed with iodized oil, located distant to the Onyx cast, do not influence the degree of artifacts generated by Onyx, and included CBCT images of patients who were additionally treated with cyanoacrylates mixed with iodized oil.
To date, there are only a limited number of scientific reports available investigating the software-based reduction of LEArelated artifacts. ${ }^{19-21}$ Lehti et al assessed the degree of artifact reduction in aortic CT angiography after Onyx 18 embolization in patients with endovascular aneurysm repair by the iterative metal artifact reduction (iMAR) software (Siemens Healthineers, Erlangen, Germany). Their study included 12 patients and demonstrated that the iMAR software can reduce the Onyx 18-related artifacts significantly. ${ }^{19}$ A further study by Stidd et al applied a prototype of a metal artifact reduction algorithm to post-interventional cerebral CBCT images of three patients with AVM who were treated with Onyx 18. They found that the investigated algorithm prototype was not effective at reducing the LEA-related artifacts. ${ }^{20}$ At present, there is one study available which investigated the software-based reduction of LEArelated artifacts in patients suffering from dAVFs. ${ }^{21}$ In the study of Otsuka et al six patients with dAVFs who were treated with Onyx were analyzed, and there was no sufficient improvement of the image quality after MAR using qualitative analysis on a four-point scale. There was no standardized quantitative analysis performed. ${ }^{21}$

For the MAR software which was used in our study, several studies demonstrated its efficacy at reducing artifacts generated by metallic implants, for example, by orthopedic metallic implants or intracranial platinum coils. ${ }^{20-24}$ The assessment of LEA-related artifact reduction including in vitro and in vivo datasets has not been in the focus of research until now. The MAR software uses an iterative metal artifact correction approach to improve the quality of CBCT imaging data and represents an extension of the algorithm first published by Prell et al which was further developed. ${ }^{30}$ From the acquired imaging data, an uncorrected volume image is reconstructed, and metal or any highly attenuating objects (in the following paragraph referred to as 'metal') are segmented, resulting in a binary metal volume image. For each projection, this binary volume is forwardprojected to yield a binary projection image of metal regions on the detector in any position. After smoothing the data along the boundary line, the projection data contained in these metal regions is replaced by a non-linear interpolation procedure. The initially corrected volume is used for a second normalized MAR correction step, including additional iterative improvements of the metal region boundaries to enhance the consistency of the corrected data. In a final step, a procedure minimizing the total variation is applied to reduce residual streaks. ${ }^{20} 22$ For this algorithm, the present study demonstrates for the first time a significant improvement of image quality after embolization with the LEA Onyx.

Previous studies on artifact suppression by LEAs only included a limited number of patients, while the present study features a combination of 10 in vitro and 20 in vivo CBCT datasets. ${ }^{19} 20$ The inclusion of an in vitro model in combination with patient data offers the advantage of a systematic analysis with an increased informative value. While the investigation of the in vitro tube models provides a high level of standardization, such as size and volume of the Onyx cast as well as the density of the surrounding brain phantom, the post-interventional CBCTs of patients with AVM or dAVF take further factors of clinical practice into account. For example, our findings indicate that a possible influence by different configurations of the individual LEA cast is limited. Moreover, the consistent analysis by setting three ROIs of the same configuration and similar location on the same image layers of the original (no MAR) and the post-processed (with MAR) CBCT images offers the advantage of a reliable comparison 
with limited susceptibility to errors. This procedure, along with the calculation of the SD of the DU, allows the consideration of Onyx-related artifacts of high and low density with the benefit that the artifacts do not cancel out each other, as might be the case for the mean density values. ${ }^{14}$ To reduce further bias, different DMSO-compatible tubes were tested before the preparation of the in vitro models and the tubes with the lowest radiopacity were used. Due to the increased informative value, qualitative analysis was carried out in addition to the standardized quantitative analysis.

An additional analysis was performed outside the region of the LEA cast in the post-interventional CBCTs of the patients with AVM or dAVF. While our findings demonstrate that the MAR software can effectively reduce the artifacts produced by Onyx, it is conceivable that the MAR software negatively influences the image quality in regions which are not affected by artifacts. This phenomenon was, however, not observed in our study. We could not detect a significant effect of the MAR software on the brain parenchyma on image layers which were initially free of LEA-related artifacts.

We acknowledge that this study has several limitations. The used tubes of the in vitro models were slightly radiopaque, which might influence the degree of artifacts and thus the process of artifact reduction. Moreover, there was no continuous flush with saline during the Onyx injection procedure of tube models, which may further affect the degree of artifacts. Eight of the 20 included post-interventional CBCTs of patients received endovascular treatment with Onyx 18 in combination with cyanoacrylates mixed with iodized oil. A possible influence on the degree of artifacts and thus the artifact reduction cannot be ruled out with certainty. Furthermore, all ROIs were drawn manually which might lead to certain bias.

\section{CONCLUSION}

The MAR software is able to reduce the artifacts evoked by Onyx in CBCT significantly without affecting the image layers free of LEA-related artifacts. Applying the MAR software could thus improve the accuracy of peri- and post-procedural CBCT imaging in clinical practice and provides the opportunity to recognize complications such as periprocedural hemorrhage promptly within the angiography suite.

Contributors NSCH, MB, MAM and DFV initiated the project. NSCH and DFV led the research, conducted the retrospective data acquisition, statistical analysis and wrote the manuscript. MB, MAM and DFV were involved in the study design and concept. CSW, LW and CMS participated in data acquisition. All authors discussed the results, commented on the paper, and approved the final version of the manuscript.

Funding The authors have not declared a specific grant for this research from any funding agency in the public, commercial or not-for-profit sectors.

\section{Competing interests None declared.}

Patient consent for publication Not required.

Ethics approval Data collection and analysis were approved by the local ethics committee (Medical Faculty of Heidelberg University; ID of the approval: S-247/2009). Patient consent was waived due to the retrospective, descriptive character of this single-center study.

Provenance and peer review Not commissioned; externally peer reviewed.

Data availability statement All data relevant to the study are included in the article or uploaded as supplemental information.

Supplemental material This content has been supplied by the author(s). It has not been vetted by BMJ Publishing Group Limited (BMJ) and may not have been peer-reviewed. Any opinions or recommendations discussed are solely those of the author(s) and are not endorsed by BMJ. BMJ disclaims all liability and responsibility arising from any reliance placed on the content. Where the content includes any translated material, BMJ does not warrant the accuracy and reliability of the translations (including but not limited to local regulations, clinical guidelines, terminology, drug names and drug dosages), and is not responsible for any error and/or omissions arising from translation and adaptation or otherwise.

Open access This is an open access article distributed in accordance with the Creative Commons Attribution Non Commercial (CC BY-NC 4.0) license, which permits others to distribute, remix, adapt, build upon this work non-commercially, and license their derivative works on different terms, provided the original work is properly cited, appropriate credit is given, any changes made indicated, and the use is non-commercial. See: http://creativecommons.org/licenses/by-nc/4.0/.

\section{ORCID iDs}

Niclas Schmitt http://orcid.org/0000-0003-2509-5459

Charlotte S Weyland http://orcid.org/0000-0002-1374-7854

Lena Wucherpfennig http://orcid.org/0000-0002-2280-1269

Dominik F Vollherbst http://orcid.org/0000-0002-8992-4757

\section{REFERENCES}

1 Gross BA, Du R. Diagnosis and treatment of vascular malformations of the brain. Curr Treat Options Neurol 2014;16:279.

2 Gross BA, Albuquerque FC, Moon K, et al. Evolution of treatment and a detailed analysis of occlusion, recurrence, and clinical outcomes in an endovascular library of 260 dural arteriovenous fistulas. J Neurosurg 2017;126:1884-93.

3 Friedlander RM. Clinical practice. arteriovenous malformations of the brain. N Eng/ J Med 2007;356:2704-12.

4 Elsenousi A, Aletich VA, Alaraj A. Neurological outcomes and cure rates of embolization of brain arteriovenous malformations with n-butyl cyanoacrylate or Onyx: a meta-analysis. J Neurointerv Surg 2016:8:265-72.

5 Schmitt N, Floca RO, Paech D. Imaging artifacts of nonadhesive liquid embolic agents in conventional and cone-beam CT in a novel in vitro AVM model. Clin Neuroradiol 2021;356.

6 Schmitt N, Floca RO, Paech D, et al. Imaging artifacts of liquid embolic agents on conventional CT in an experimental in vitro model. AJNR Am J Neuroradiol 2021:42:126-31.

7 Vollherbst DF, Otto R, Do T, et al. Imaging artifacts of Onyx and PHIL on conventional CT, cone-beam CT and MRI in an animal model. Interv Neuroradiol 2018;24:693-701.

8 Pop R, Mertz L, llyes A, et al. Beam hardening artifacts of liquid embolic agents: comparison between Squid and Onyx. J Neurointerv Surg 2019;11:706-9.

9 Lv X, Wu Z, Li Y, et al. Hemorrhage risk after partial endovascular NBCA and ONYX embolization for brain arteriovenous malformation. Neurol Res 2012;34:552-6.

10 Gross BA, Albuquerque FC, McDougall CG, et al. A multi-institutional analysis of the untreated course of cerebral dural arteriovenous fistulas. J Neurosurg 2018:129:1114-9.

11 Pereira GC, Traughber M, Muzic RF. The role of imaging in radiation therapy planning: past, present, and future. Biomed Res Int 2014;2014:231090.

12 Shtraus N, Schifter D, Corn BW, et al. Radiosurgical treatment planning of AVM following embolization with Onyx: possible dosage error in treatment planning can be averted. J Neurooncol 2010;98:271-6.

13 Roberts DA, Balter JM, Chaudhary N, et al. Dosimetric measurements of Onyx embolization material for stereotactic radiosurgery. Med Phys 2012;39:6672-81.

14 Giantsoudi D, De Man B, Verburg J, et al. Metal artifacts in computed tomography for radiation therapy planning: dosimetric effects and impact of metal artifact reduction. Phys Med Biol 2017;62:R49-80.

15 Hasegawa H, Hanakita S, Shin M, et al. Integration of rotational angiography enables better dose planning in gamma knife radiosurgery for brain arteriovenous malformations. J Neurosurg 2018;129:17-25.

16 Heran NS, Song JK, Namba K, et al. The utility of DynaCT in neuroendovascular procedures. AJNR Am J Neuroradiol 2006;27:330-2.

17 Psychogios M-N, Buhk J-H, Schramm P, et al. Feasibility of angiographic CT in periinterventional diagnostic imaging: a comparative study with multidetector CT. AJNR Am J Neuroradiol 2010;31:1226-31.

18 Katsura M, Sato J, Akahane M, et al. Current and novel techniques for metal artifact reduction at CT: practical guide for radiologists. Radiographics 2018:38:450-61.

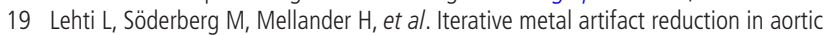

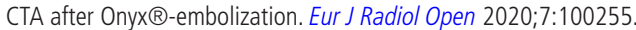

20 Stidd DA, Theessen H, Deng Y, et al. Evaluation of a metal artifacts reduction algorithm applied to postinterventional flat panel detector CT imaging. AJNR Am J Neuroradiol 2014:35:2164-9.

21 Otsuka T, Nishihori M, Izumi T, et al. Streak metal artifact reduction technique in cone beam computed tomography images after endovascular neurosurgery. Neurol Med Chir 2021;61:468-74.

22 Meyer E, Raupach $\mathrm{R}$, Lell M, et al. Normalized metal artifact reduction (NMAR) in computed tomography. Med Phys 2010;37:5482-93.

23 Cay C, Wells M, Wang A. Quantitative assessment of metal artifact reduction in C-arm cone-beam CT guidance of neurovascular interventions $2013 \mathrm{https}: / / \mathrm{www}$. researchgate.net/publication/266096798_Quantitative_Assessment_of_Metal_ Artifact Reduction_in_C-arm Cone-beam_CT Guidance of Neurovascular Interventions 
24 Psychogios M-N, Scholz B, Rohkohl C, et al. Impact of a new metal artefact reduction algorithm in the noninvasive follow-up of intracranial clips, coils, and stents with flat-panel angiographic cta: initial results. Neuroradiology 2013;55:813-8.

25 Saeki N, Rhoton AL. Microsurgical anatomy of the upper basilar artery and the posterior circle of Willis. J Neurosurg 1977;46:563-78.

26 Daubner D, Spieth S, Cerhova J, et al. Measuring ventricular width on cranial computed tomography: feasibility of dose reduction in a custom-made adult Phantom. Rofo 2016;188:73-81.
27 Nolden M, Zelzer S, Seitel A, et al. The Medical Imaging Interaction Toolkit: challenges and advances : 10 years of open-source development. Int J Comput Assist Radio/ Surg 2013:8:607-20.

28 Cohen J. A coefficient of agreement for nominal scales. Educ Psychol Meas 1960;20:37-46.

29 McHugh ML. Interrater reliability: the kappa statistic. Biochem Med 2012;22:276-82.

30 Prell D, Kyriakou Y, Beister M, et al. A novel forward projection-based metal artifact reduction method for flat-detector computed tomography. Phys Med Biol 2009;54:6575-91. 